\title{
A Kalman filtering induced heuristic optimization based partitional data clustering
}

\author{
Arjun Pakrashi ${ }^{\mathrm{a}, *}$, Bidyut B. Chaudhuri ${ }^{\mathrm{b}}$ \\ ${ }^{a}$ Insight Centre for Data Analytics, University College Dublin, Ireland \\ ${ }^{b}$ Computer Vision 83 Pattern Recognition Unit, Indian Statistical Institute, 203 B.T. Road, \\ Kolkata 700108, India
}

\begin{abstract}
Clustering algorithms have regained momentum with recent popularity of data mining and knowledge discovery approaches. To obtain good clustering in reasonable amount of time, various meta-heuristic approaches and their hybridization, sometimes with K-Means technique, have been employed. A Kalman Filtering based heuristic approach called Heuristic Kalman Algorithm (HKA) has been proposed a few years ago, which may be used for optimizing an objective function in data/feature space. In this paper at first HKA is employed in partitional data clustering. Then an improved approach named HKA-K is proposed, which combines the benefits of global exploration of HKA and the fast convergence of K-Means method. Implemented and tested on several datasets from UCI machine learning repository, the results obtained by HKA-K were compared with other hybrid meta-heuristic clustering approaches. It is shown that HKA-K is atleast as good as and often better than the other compared algorithms.
\end{abstract}

Keywords: Clustering, K-Means, Optimization, Metaheuristic Optimization, Heuristics

\section{Introduction}

Clustering is the process of assigning a set of $n$ data points into $C$ classes based on the similarity between the data points in the feature space. It is useful when some prototype data from known classes are not available for training a supervised classifier or for an exploratory data analysis task. It is one of the earliest pattern classification approaches and has found renewed interest since the beginning of data mining and big data analytics.

\footnotetext{
${ }^{*}$ Corresponding Author

Email addresses:

arjun.pakrashi@ucdconnect.ie, arjun.pakrashi@insight-centre.org (Arjun Pakrashi), bbc@isical.ac.in (Bidyut B. Chaudhuri)
}

Preprint submitted to Elsevier 
Clustering can be broadly classified into two categories: Hierarchical and Partitional. Hierarchical clustering methods are again grouped into two types, namely divisive or top-down and agglomerative or bottom-up methods. If the number of desired clusters is known (say, $K$ ) a priori, the approach can be made non-hierarchical and the data can be assigned into $K$ clusters using a partitional clustering algorithm. Else, several clusterings are generated and the best among them is chosen on the basis of some objective criterion.

One of the classical partitional clustering approaches is the K-Means algorithm, which is a simple way to discover convex, especially hyper-spherical clusters. It starts with $K$ seed points in the feature space representing the initial cluster centers. Then it assigns a data point $p$ to the cluster whose center is nearest to $p$. When all data are assigned in such a way, the centers are updated by the mean position of current data assigned to each cluster. Then a new iteration starts and the data are redistributed using the nearness criterion stated above. The process is repeated till the centroids do not change anymore or a specified number of iterations is reached.

However, K-Means approach may fail to find desired results for some choice of initial seed points. In such a case it converges to a local sub-optimal solution, or may even fail to converge [38]. One of the various techniques proposed to tackle the problem model the clustering task as a non-convex optimization problem employing a cluster quality based objective function and then try to find its global optimum. Since finding such an optimum is an NP-Complete problem [40], metaheuristic algorithms are employed, among others, to find a near optimal solution. A metaheuristic algorithm only uses values of the objective function to be optimised and does not require the function to have derivatives. Hence, metaheuristic algorithms are one of the choices to optimize the non-convex optimization problems. Examples of metaheuristic based methods are PSO (Particle Swarm Optimization), GA (Genetic Algorithm), ACO (Ant Colony Optimization) etc. Another class of algorithms are based on hybrid approach, where two or more metaheuristic algorithms are combined, or metaheuristic algorithms are combined with K-Means or other local search methods. Some examples of this category are hybrid of K-Means and PSO, hybrid of K-Means and GA as well as PSO-ACO-K-Means hybrid algorithms. A review on different clustering techniques can be found in [18], while a survey of metaheuristic algorithms is given in [30]. A brief survey of metaheuristic based clustering algorithms will be presented in Section 2 of this paper.

A new metaheuristic based optimization approach named Heuristic Kalman Algorithm (HKA) has been proposed by Toscano and Lyonnet in [41]. This method uses a random number generator with Gaussian probability distribution to choose a set of points in the search space. At each subsequent iteration, the mean vector and covariance matrix for the Gaussian random number generator are modified using a Kalman filtering framework, so that the mean gets nearer to the global optimum.

In the past, HKA has been employed in applied problems like tuning of PID controllers [41] and in robust observer for rotor flux estimation of induction machine [42]. The present paper uses HKA for data clustering context. More 
specifically, the contributions of this paper are as follows.

- To employ HKA for data clustering and show that it performs well on synthetic and real datasets.

- To propose an improvement over the above HKA based approach through hybridization with a K-Means like approach. The proposed approach is named HKA-K where the $\mathrm{K}$ indicates application of K-Means like approach. It is demonstrated that HKA-K converges faster than HKA.

- To demonstrate that the performance of HKA-K in terms of accuracy, convergence and computational efficiency is as good as or better than several other state of the art metaheuristic based clustering algorithms.

The rest of the paper is organized as follows. We briefly survey the metaheuristic algorithms and hybrid metaheuristic algorithms for data clustering in Section 2 . In Section 3 the HKA based optimization approach is explained by following the paper [41]. A brief exposition of partitional clustering, along with centroid based $\mathrm{K}$-Means approach is provided in Section 4 The approach of using HKA to data clustering is introduced in the same section. Then the proposed improvement on HKA clustering, namely HKA-K approach is developed in Section 5. Experimental results are presented and discussed in Section [6 where comparisons are also made with other metaheuristic based methods. Section 7 contains some concluding remarks on the paper.

\section{Brief survey on metaheuristic based clustering}

Metaheuristic population based optimization algorithms in general are used in clustering either as is, or as a hybrid variant combining multiple algorithms. In general two or more population based algorithm and/or a local search algorithm are combined together.

Among pure and hybrid metaheuristic based clustering approaches, a Genetic Algorithm (GA) based approach named COWCLUS 9] was presented, which uses Calinski and Harabasz Variance Ratio Criteria [5] as the cost function and performs standard GA to optimize it. Another approach in 29] has used GA to optimize the clustering objective function. Yet another GA based approach named GKA [23] has introduced a new mutation operator and combined it with K-Means for better convergence. These early methods consider each chromosome representing a clustering of the data points. The chromosome has $n$ alleles, each taking an integer value corresponding to one particular cluster. On the other hand, the GA based method KGA [27] encodes only the cluster centers in the chromosomes as real numbers. In this case if $K$ clusters are to be obtained on $d$-dimensional data, each chromosome will have a length of $(K . d)$. This method combines K-Means and GA and is more effective than the previous ones. A Differential Evolution (DE) and K-Means based algorithm 
was proposed in [26], where the K-Means method is used to refine the results given by the recombination operators of $\mathrm{DE}$.

Particle Swarm Optimization (PSO) has also been employed for data clustering [34, 19, 32]. PSO was hybridized with K-Means in [2, 10, 43, 34] to improve convergence. In [46] a Gaussian based distance metric [45] was used while clustering the data using PSO algorithm. Also, an approach combining GA and PSO is given in [25]. Another hybrid algorithm 24] employs GA, PSO and K-Means approach to determine the number of clusters as well as to perform clustering.

Among other metaheuristic approaches, Artificial Bee Colony (ABC) optimization 20, 21] was applied for clustering data 22, 47|. Also, an Ant Colony Optimization (ACO) based technique was proposed in [39]. In 31] an approach involving PSO, ACO and K-Means while in [33] an ACO and Simulated Annealing (SA) based algorithm was proposed. The swarm based approach with honey-bee mating process named Honey Bee Mating Optimization (HBMO) [1] was used in clustering domain [14].

Clustering was done with Artificial Fish Swarm (AFS) algorithm [48, 7] and Bacteria Foraging Algorithm (BFO) 44] as well. Clustering using Cuckoo Search approach was also proposed in [36]. Moreover, an automatic clustering using Invasive Weed Optimization (IWO) can be found in [8]. A new method, Gravitational Search Algorithm (GSA), proposed in [35], was used to cluster data [16]. Another approach called Black-Hole search, was also applied in data clustering [15].

In the following section we briefly introduce HKA and then explain how it can be used for clustering.

\section{Heuristic Kalman Algorithm (HKA)}

The HKA [41] is a non-convex, population-based, metaheuristic optimization algorithm, which works in a manner different from other population based metaheuristic approaches. Let an objective function $J$ be defined over a multidimensional and bounded real space $S$. We have to find the position of a point in $S$ where the value of $J$ is optimum. The HKA based approach in [41] for finding this point is described below.

A solution is generated through a Gaussian probability density function (pdf), where the mean vector represents the position in $S$ and the variance matrix represents the uncertainty of the solution. Given a search space $S$, the objective of the algorithm is to modify the mean $\boldsymbol{m}^{(k)}$ and variance matrix $\boldsymbol{P}^{(k)}$ of a Gaussian pdf at $k^{\text {th }}$ iteration such that the mean ideally approaches the global optima within $S$ with respect to the objective function $J$, along with decreasing variance. The modification of the mean and variance matrix is done by an approach based on Kalman filtering. The algorithm starts with an initial mean vector $\boldsymbol{m}^{(0)}$ and variance matrix $\boldsymbol{P}^{(0)}$. The computation of the initial values are explained later. At each $k^{\text {th }}$ iteration the measurement step generates $N$ number of points given by vectors $\boldsymbol{x}(k)=\left\{\boldsymbol{x}_{1}^{(k)}, \boldsymbol{x}_{2}^{(k)}, \ldots, \boldsymbol{x}_{N}^{(k)}\right\}$ from a Gaussian pdf 
$\mathcal{N}\left(\boldsymbol{m}^{(k)}, \boldsymbol{P}^{(k)}\right)$, which are ordered such that $J\left(\boldsymbol{x}_{1}^{(k)}\right) \leq J\left(\boldsymbol{x}_{1}^{(k)}\right) \leq \ldots \leq J\left(\boldsymbol{x}_{N}^{(k)}\right)$ is satisfied. Another Gaussian pdf $\mathcal{N}\left(\boldsymbol{\xi}^{(k)}, \boldsymbol{V}^{(k)}\right)$ is generated by taking the mean $\boldsymbol{\xi}^{(k)}$ (Eq. (1)) of the vector positions of top $N_{\xi}$ number of points, and the variance $\boldsymbol{V}^{(k)}$ (Eq. (2)) is computed on these top $N_{\xi}$ points. This is considered to be the measurement. Next, in the estimation step, the Kalman filter is used to combine these two Gaussian pdfs to generate a new Gaussian pdf $\mathcal{N}\left(\boldsymbol{m}^{(k+1)}, \boldsymbol{P}^{(k+1)}\right)$, where $\boldsymbol{m}^{(k+1)}$ is expected to be closer to the desired solution than $\boldsymbol{m}^{(k)}$. Here $\boldsymbol{m}^{(k+1)}$ is computed using Eq. (3), (4) and (5) while $\boldsymbol{P}^{(k+1)}$ is computed using Eq. (5), (6), (77) and (8). If a stopping condition is encountered, then it takes the so far found best value of $\boldsymbol{m}^{(k)}$, else it carries the Gaussian pdf computed for the estimation step in the next iteration and repeats the process.

It should be mentioned that, in the current formulation, the matrices $\boldsymbol{V}^{(k)}$ and $\boldsymbol{P}^{(k)}$ contain only the diagonal elements and hence can also be treated as vector of dimension $d$, which is the dimension of the feature space $S$.

The value of $\boldsymbol{\xi}^{(k)}$ is calculated as

$$
\boldsymbol{\xi}^{(k)}=\frac{1}{N_{\xi}} \sum_{i=1}^{N_{\xi}} \boldsymbol{x}_{i}^{(k)}
$$

Also, the uncertainty matrix $\boldsymbol{V}^{(k)}$ is given by

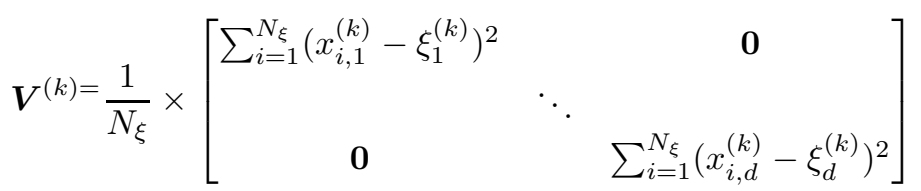

The estimation step computes the parameters $\boldsymbol{m}^{(k+1)}, \boldsymbol{P}^{(k+1)}$ for Gaussian pdf for next iteration by

$$
\begin{gathered}
\hat{\boldsymbol{m}}^{(k)}=\boldsymbol{m}^{(k)}+\boldsymbol{L}^{(k)}\left(\boldsymbol{\xi}^{(k)}-\boldsymbol{m}^{(k)}\right) \\
\boldsymbol{m}^{(k+1)}=\hat{\boldsymbol{m}}^{(k)} \\
\boldsymbol{L}^{(k)}=\boldsymbol{P}^{(k)}\left(\boldsymbol{P}^{(k)}+\boldsymbol{V}^{(k)}\right)^{-1} \\
\hat{\boldsymbol{P}}^{(k)}=\left(\boldsymbol{I}-\boldsymbol{L}^{(k)}\right) \boldsymbol{P}^{(k)} \\
\boldsymbol{P}^{(k+1)}=\left(\sqrt{\boldsymbol{P}^{(k)}}+a^{(k)}\left(\sqrt{\hat{\boldsymbol{P}}^{(k)}}-\sqrt{\boldsymbol{P}^{(k)}}\right)\right)^{2}
\end{gathered}
$$

Here $\hat{\boldsymbol{P}}^{(k)}$ is the predicted covariance matrix and $\boldsymbol{L}^{(k)}$ is the Kalman gain. The details of Kalman filter and Kalman gain can be found in [28, 13]. A 
slowdown factor $a^{(k)}$ is introduced here to control the fast decrease of the covariance matrix, which otherwise could converge prematurely to a bad solution. The factor is defined as

$$
a^{(k)}=\frac{\alpha \cdot \min \left(1,\left(\frac{1}{d} \sum_{i=1}^{d} \sqrt{v_{i, i}^{(k)}}\right)^{2}\right)}{\min \left(1,\left(\frac{1}{d} \sum_{i=1}^{d} \sqrt{v_{i, i}^{(k)}}\right)^{2}\right)+\max _{1 \leq i \leq d}\left(\sqrt{\hat{p}_{i, i}^{(k)}}\right)}
$$

In $\mathrm{Eq}(8), \alpha$ is the slowdown co-efficient, which is a parameter of the algorithm, and $v_{i, i}^{(k)}$ and $\hat{p}_{i, i}^{(k)}$ are the $i^{t h}$ diagonal of the matrices $\boldsymbol{V}^{(k)}$ and $\hat{\boldsymbol{P}}^{(k)}$ respectively.

The process is repeated till the value of $k$ reaches a predefined number, or the best $N_{\xi}$ solutions generated at some iteration is within a hypersphere with a predefined radius $\rho$, centered about the best of the generated vectors $\boldsymbol{x}_{1}^{(k)}$. In both cases, the found best $\boldsymbol{m}^{(k)}$ is declared as the solution.

The initial value of $\boldsymbol{m}^{(0)}$ and $\boldsymbol{P}^{(0)}$ in HKA are computed as follows.

$$
\begin{aligned}
m_{i}^{(0)} & =\frac{\max \left(S_{i}\right)+\min \left(S_{i}\right)}{2} \\
p_{i, i}^{(0)} & =\frac{\max \left(S_{i}\right)-\min \left(S_{i}\right)}{6}
\end{aligned}
$$

Here $m_{i}^{(0)}$ is the $i^{\text {th }}$ component of the mean vector, $p_{i, i}^{(0)}$ is the $i^{\text {th }}$ diagonal of the variance matrix, while $\max \left(S_{i}\right)$ and $\min \left(S_{i}\right)$ indicate the maximum and minimum bound value along the $i^{t h}$ dimension of the search space $S$.

The convergence properties and other analysis of HKA can be found in [4] where the authors have shown that HKA performs well with respect to several test functions and attains better results than other algorithms most of the time. The authors of HKA in [41] have also shown that the number of function evaluations to reach the solution for HKA is lower than a few other metaheuristic optimization algorithms and this method was able to reach better results most of the time. Also, constrained optimization was demonstrated by the authors in solving the welded beam design problem and in tuning Proportional Integral Derivative (PID) controllers.

\section{Partitional clustering using HKA}

\subsection{Partitional Clustering}

Assume a dataset $\boldsymbol{X}=\left\{\boldsymbol{x}_{1}, \boldsymbol{x}_{2}, \ldots, \boldsymbol{x}_{n}\right\}$, where $\boldsymbol{x}_{i}, 1 \leq i \leq n$ is a datum represented as a vector in $d$-dimensional feature space. The task of clustering is to partition $\boldsymbol{X}$ into a set of $K$ clusters $\boldsymbol{C}=\left\{\boldsymbol{c}_{1}, \boldsymbol{c}_{2}, \ldots, \boldsymbol{c}_{K}\right\}$ such that cluster $\boldsymbol{c}_{i}$ contains data points similar to each other and dissimilar to the data of other clusters with respect to some measure of similarity. In crisp partitional clustering all clusters should be non-empty $\forall_{1 \leq j \leq K}\left(\boldsymbol{c}_{j} \neq \phi\right)$, and having no 
common elements $\boldsymbol{c}_{j} \cap \boldsymbol{c}_{m}=\phi$ where $1 \leq j \leq K, 1 \leq m \leq K$ and $j \neq m$ while $\cup_{j=1}^{K} \boldsymbol{c}_{j}=\boldsymbol{X}$.

The centroids for the set $\boldsymbol{C}$ are $\boldsymbol{Z}=\left\{\boldsymbol{z}_{1}, \boldsymbol{z}_{2}, \ldots, \boldsymbol{z}_{k}\right\}$ where $\boldsymbol{z}_{i}$ is given by Eq. (11)

$$
\boldsymbol{z}_{j}=\frac{1}{\operatorname{card}\left(\boldsymbol{c}_{j}\right)} \sum_{\boldsymbol{x}_{i} \in \boldsymbol{c}_{j}} \boldsymbol{x}_{i}
$$

where $1 \leq j \leq K$, and $\operatorname{card}($.$) is the cardinality of the set (.) .$

Several algorithms consider clustering as a global optimization problem, where one or more objective functions representing the cluster quality is optimized. Here we have used the sum of squared intra-cluster Euclidean distances as the objective function to minimize, which is given by

$$
J(\boldsymbol{Z})=\sum_{\boldsymbol{z}_{j} \in \boldsymbol{Z}} \sum_{\boldsymbol{x}_{i} \in \boldsymbol{X}} \mu_{i j}\left\|\boldsymbol{x}_{i}-\boldsymbol{z}_{j}\right\|^{2}
$$

Where $\|$.$\| is the magnitude of the vector (.) in Euclidean space, and \mu_{i j}$ is the cluster assignment value defined as

$$
\mu_{i j}= \begin{cases}1 & \text { if } \boldsymbol{x}_{i} \in \boldsymbol{c}_{j} \\ 0 & \text { otherwise }\end{cases}
$$

where $1 \leq i \leq n$ and $1 \leq j \leq K$.

Therefore the objective is to optimize Eq. (12) with respect to $\boldsymbol{Z}$, on a fixed dataset $\boldsymbol{X}$, and find the cluster assignment $\mu_{i j}$ for all $i$ and $j$.

Since we have used the "idea" of K-Means algorithm in our HKA-K approach, a brief description of K-Means algorithm is given below.

1. The number of clusters $K$ is prespecified. Initially, the set of cluster centers $\boldsymbol{Z}$ is randomly generated.

2. A data point $\boldsymbol{x}_{i}$ is assigned to $\boldsymbol{c}_{j}$ if $\boldsymbol{z}_{j}$ is closest to $\boldsymbol{x}_{i}$, ie. $\left\|\boldsymbol{x}_{i}-\boldsymbol{z}_{j}\right\| \leq$ $\left\|\boldsymbol{x}_{i}-\boldsymbol{z}_{m}\right\|$, where $1 \leq m \leq K$ and $j \neq m$. All datapoints are assigned in this way.

3. Using Eq. (11), the cluster center $\boldsymbol{z}_{j}$ is updated with the datapoints assigned to the $j^{\text {th }}$ cluster in Step 2. All the cluster centers are updated in this way.

4. If a termination criterion is satisfied, then the process is stopped. Else, the control goes to Step 2 with current cluster centers.

A detailed analysis of K-Means approach can be found in [4].

\subsection{HKA based partitional clustering}

For our purpose we represent the cluster centers as a concatenated vector.

If the $K$ cluster centers are $\boldsymbol{z}_{1}, \boldsymbol{z}_{2}, \ldots, \boldsymbol{z}_{K}$ in $\mathbb{R}^{d}$, then the concatenated vector will be $\boldsymbol{q}=\left[\boldsymbol{z}_{1}, \boldsymbol{z}_{2}, \ldots, \boldsymbol{z}_{K}\right]$, where [.] denotes the concatenation of the comma 
separated vectors. Clearly, the dimension of vector $\boldsymbol{q}$ will be $K$.d. To compute the objective value for such a concatenated vector, the individual $\boldsymbol{z}_{i}$ s are extracted from $\boldsymbol{q}$ and Eq. (12) is used.

The clustering process progresses as in the HKA algorithm. It starts with an initial mean $\boldsymbol{m}^{(0)}$ and covariance matrix $\boldsymbol{P}^{(0)}$ for the Gaussian random number generator. $\boldsymbol{m}_{\text {best }}^{(0)}$ is set to $\boldsymbol{m}^{(0)}$. In every iteration $k$, the process generates $N$ random vectors from a Gaussian pdf with mean vector and covariance matrix $\boldsymbol{m}^{(k)}$ and $\boldsymbol{P}^{(k)}$, respectively. Next, it computes $\boldsymbol{\xi}^{(k)}$ using Eq. (10) and $\boldsymbol{V}^{(k)}$ using Eq. (2) and then estimates $\hat{\boldsymbol{m}}^{(k)}$ using Eq. (3) and $\hat{\boldsymbol{P}}^{(k)}$ using Eq. (6) and (5). If the objective value of $\hat{\boldsymbol{m}}^{(k)}$ is better than $\boldsymbol{m}_{\text {best }}^{(k)}$ then it is updated with $\hat{\boldsymbol{m}}^{(k)}$. For the next iteration, $\boldsymbol{m}^{(k+1)}$ and $\boldsymbol{P}^{(k+1)}$ are computed as in Eq. (44) and Eq. (7) respectively, and $k$ is incremented. This process is repeated until the value of $k$ reaches some prefixed number of iterations, or the best $N_{\xi}$ samples generated in some iteration is within a hypersphere of a predefined radius $\rho$, centered at the best sample point. When terminated, $\boldsymbol{m}_{\text {best }}^{(k)}$ is taken as the solution. Here, $\boldsymbol{m}_{\text {best }}, \hat{\boldsymbol{m}}, \boldsymbol{\xi}$ and the generated vectors from the Gaussian pdfs are encoded as described in the previous paragraph.

For initialization, $\boldsymbol{m}^{(0)}$ is chosen in a similar way as in HKA but here the search space is considered as the minimum bounding hyperbox of the dataset.

$$
\boldsymbol{m}^{(0)}=\operatorname{cat}(\overline{\boldsymbol{x}}, K) ; \bar{x}_{j}=\frac{\max _{X}\left(\boldsymbol{x}_{j}\right)+\min _{X}\left(\boldsymbol{x}_{j}\right)}{2} ; 1 \leq j \leq d
$$

Here $\operatorname{cat}(\boldsymbol{v}, K)$ is the concatenation of the vector $\boldsymbol{v}, K$ times. $\max _{X}\left(\boldsymbol{x}_{j}\right)$ (and $\min _{X}\left(\boldsymbol{x}_{j}\right)$ ) denotes the maximum (and minimum) value of dimension $j$ over all datapoints in the dataset.

The initial covariance matrix $\boldsymbol{P}^{(0)}$ is taken as

$$
\begin{gathered}
\boldsymbol{P}^{(0)}=\left[\begin{array}{ccc}
\left(\sigma_{1}^{(0)}\right)^{2} & \cdots & \mathbf{0} \\
\vdots & \ddots & \vdots \\
\mathbf{0} & \cdots & \left(\sigma_{(K . d)}^{(0)}\right)^{2}
\end{array}\right] \\
\boldsymbol{\sigma}^{(0)}=\operatorname{cat}(\tilde{\boldsymbol{x}}, K) ; \tilde{x}_{j}=\frac{\max _{X}\left(\boldsymbol{x}_{j}\right)-\min _{X}\left(\boldsymbol{x}_{j}\right)}{6} ; 1 \leq j \leq d
\end{gathered}
$$

Given a dataset, the minimum bounding hyperbox will contain all the data points inside the hyperbox. As we are performing a centroid based clustering task and the cluster centers cannot be outside this bounding box, the algorithm does not need to search outside this hyperbox. Therefore we select the starting point of the search at the center of the minimum bounding hyperbox and select a variance which is sufficiently large so that the random samples covers almost the entire search space. Also, we limit the search so that the search does not cross this hyperbox. 


\section{HKA-K Clustering}

Though HKA performs fairly well for clustering data, as shown in Section 6. it would be interesting to see if the results could be improved. Improvement can be envisioned on two aspects, namely accuracy of the results and efficiency of the algorithm. One possible way of achieving both is to exploit global search nature of the algorithm combined with a local search. We have tried to attain this by exploiting the simplicity and speed of K-Means approach under a hybrid framework. A centroid based K-Means like step is used because of its simplicity. A full K-Means algorithm is not run, instead we only use one single step of centroid updates with the K-Means approach at each iteration.

In this work we make the following two modifications: (a) we introduce a new step after the estimation step of HKA with a view to attain faster convergence and better accuracy, (b) we incorporate a restart mechanism which in effect attempts to avoid the search getting trapped in a local optimum or stagnation. We show that these modifications are effective in improving convergence and speed compared to HKA based clustering as well as several other state-of-theart metaheuristic based clustering algorithms.

For the above process, we introduce a time update step which updates the estimated $\hat{\boldsymbol{m}}^{(k)}$ through one single step of weighted K-Means operator. The

estimated $\hat{\boldsymbol{P}}^{(k)}$ is also updated accordingly. See [28, 13] for time update in Kalman filtering approach. On the other hand, in HKA the estimated $\hat{\boldsymbol{m}}^{(k)}$ is directly used in the next iteration (Eq. (44)). By the term K-Means operator we mean one single iteration of K-Means which will update the centroids to move towards the solution, we control this movement through weight. We also introduce a conditional restart mechanism. The search is restarted if the $N_{\xi}$ sample points fall in a small region limited by a constant $\epsilon$.

Algorithm 1 describes HKA-K in steps. After initialization in Step 1, $N$ vectors $\boldsymbol{q}(k)=\left\{\boldsymbol{q}_{1}^{(k)}, \boldsymbol{q}_{2}^{(k)}, \ldots, \boldsymbol{q}_{N}^{(k)}\right\}$ are generated in Step 2 randomly from the Gaussian pdf at the $k^{t h}$ iteration using the mean $\boldsymbol{m}^{(k)}$ and covariance matrix $\boldsymbol{P}^{(k)}$. In this context, the mean and covariance matrix are the parameters to the Gaussian pdf which is modified by the Kalman filter framework as in HKA. These parameters should not to be confused with the mean or covariance matrix of the data set.

Next, in Step 3, the measurement $\boldsymbol{\xi}^{(k)}$ is taken by finding the mean value of the $N_{\xi}$ points as in Eq. (1) and the measurement error $\boldsymbol{V}^{(k)}$ by Eq. (2). Next Eq. (3), (5), (6) are used to get the state update $\hat{\boldsymbol{m}}^{(k)}$ and the related variance $\hat{\boldsymbol{P}}^{(k)}$ in Step 4. The time update is performed at Step 5, where at first each of the $K$ cluster centroids in $\hat{\boldsymbol{m}}^{(k)}$ are extracted from the vector representation and the K-Means operator is used to get the centers $\boldsymbol{m}^{\prime(k)}$ are obtained. Now, the centers for the next iteration is given by

$$
\boldsymbol{m}^{(k+1)}=\hat{\boldsymbol{m}}^{(k)}+w\left(\boldsymbol{m}^{(k)}-\hat{\boldsymbol{m}}^{(k)}\right)
$$

The weight $w$ used to control the influence of K-Means can be a constant or 
Algorithm 1 HKA-K

- Step 1, Select $N, N_{\xi}, \alpha, w$, maxiter and $\epsilon$. Initialize mean $\boldsymbol{m}^{(0)}$ using Eq. (13), covariance matrix $\boldsymbol{P}^{(0)}$ using Eq. (14), (15). Set $\boldsymbol{m}_{\text {best }}^{(0)}=\boldsymbol{m}^{(0)}$ and $k=0$.

- Step 2, Randomly generate $N$ number of $(K . d)$-dimensional vectors $\boldsymbol{q}(k)=\left\{\boldsymbol{q}_{1}^{(k)}, \boldsymbol{q}_{2}^{(k)}, \ldots, \boldsymbol{q}_{N}^{(k)}\right\}$ from Gaussian pdf $\mathcal{N}\left(\boldsymbol{m}^{(k)}, P^{(k)}\right)$.

- Step 3, Compute the measurement point $\boldsymbol{\xi}^{(k)}$ using equation Eq. (11) and the variance $\boldsymbol{V}^{(k)}$ using Eq. (2).

- Step 4, Compute estimation of the optimum $\hat{\boldsymbol{m}}^{(k)}$ and variance $\hat{\boldsymbol{P}}^{(k)}$ using Eq. (3), (6) and (5).

- Step 5, Apply single step of K-Means on $\hat{\boldsymbol{m}}^{(k)}$ to get $\boldsymbol{m}^{(k)}$, use Eq. (16) to get $\boldsymbol{m}^{(k+1)}$ and Eq. (18), (19) to compute $\hat{\boldsymbol{W}}^{(k+1)}$. Apply slowdown factor and adjust the time updated variance $\boldsymbol{P}^{(k+1)}$ as in Eq. (20).

- Step 6, If $J\left(\boldsymbol{m}^{\prime(k)}\right)<J\left(\boldsymbol{m}_{\text {best }}^{(k)}\right)$ then set $\boldsymbol{m}_{\text {best }}^{(k+1)}=\boldsymbol{m}^{\boldsymbol{\prime}^{(k)}}$, else set $\boldsymbol{m}_{\text {best }}^{(k+1)}=\boldsymbol{m}_{\text {best }}^{(k)}$

- Step 7, If $\rho<\epsilon$ as in Eq. (21), then reset $k=0$, Generate $\boldsymbol{m}^{(k)}$ by uniformly selecting $K$ random data points and concatenating them. Generate $\boldsymbol{P}^{(k)}$ as per Eq. (14), (15).

- Step 8, If $k>$ maxiter then terminate the algorithm. Output $\boldsymbol{m}_{\text {best }}^{(k)}$ as the solution. Else set $k=k+1$, goto Step 2

a function. For our experiments we took $w$ as a constant number in the interval $[0,1]$. A moderate value of $w$ should be selected such that the effect of HKA and K-Means are balanced.

From the Kalman filtering algorithm, ignoring the control inputs and process noise, the state and its related covariance matrix for the next iteration can be obtained as

$$
\begin{gathered}
\boldsymbol{m}^{(k+1)}=\boldsymbol{A}^{(k)} \hat{\boldsymbol{m}}^{(k)} \\
\hat{\boldsymbol{W}}^{(k+1)}=\left(\boldsymbol{A}^{(k)}\right) \hat{\boldsymbol{P}}^{(k)}\left(\boldsymbol{A}^{(k)}\right)^{T}
\end{gathered}
$$

Here $\boldsymbol{A}^{(k)}$ is the state transition matrix. The related covariance matrix is updated as shown in Eq. (18). We find $\boldsymbol{m}^{(k+1)}$ by Eq. (16) and hence to update 
the covariance matrix we need to find $\boldsymbol{A}^{(k)}$. We consider $\boldsymbol{A}^{(k)}$ to be a diagonal matrix with the components given by

$$
a_{i, i}^{(k)}=\frac{m_{i}^{(k+1)}}{\hat{m}_{i}^{(k)}} ; 1 \leq i \leq(K . d)
$$

Where $a_{i, i}^{(k)}$ is the $i^{t h}$ diagonal component of $\boldsymbol{A}^{(k)}$. Conversely in HKA, $\boldsymbol{A}^{(k)}$ is considered identity matrix and $\boldsymbol{m}^{(k+1)}$ is equal to $\hat{\boldsymbol{m}}^{(k)}$. Now $\boldsymbol{A}^{(k)}$ is used to update the variance by Eq. (18). The mean $\boldsymbol{m}^{(k+1)}$ is used in the next iteration and $\boldsymbol{P}^{(k+1)}$ is computed from $\boldsymbol{W}^{(k+1)}$ after a slowdown step in Eq. (20) is used in the next iteration with the Gaussian pdf.

In each iteration the best vector found so far $\boldsymbol{m}_{\text {best }}^{(k)}$ is retained in Step 6, which indicates the optimum value of $\boldsymbol{m}^{(k)}$ found till the current iteration. The initial value $\boldsymbol{m}_{\text {best }}^{(0)}$ is set to $\boldsymbol{m}^{(0)}$. If $J\left(\boldsymbol{m}^{(k)}\right)<J\left(\boldsymbol{m}_{\text {best }}^{(k)}\right)$ then $\boldsymbol{m}_{\text {best }}^{(k+1)}$ is set to $\boldsymbol{m}^{(k)}$. Otherwise, $\boldsymbol{m}_{\text {best }}^{(k)}$ is retained as $\boldsymbol{m}_{\text {best }}^{(k+1)}$. Note that we use the unweighted K-Means step to store the best vector, while employ weighted updating for further search.

The slowdown factor $a$ is computed as done in HKA. Therefore the variance matrix update after the modification along with slowdown becomes

$$
\boldsymbol{P}^{(k+1)}=\left(\sqrt{\boldsymbol{P}^{(k)}}+a\left(\sqrt{\boldsymbol{W}^{(k+1)}}-\sqrt{\boldsymbol{P}^{(k)}}\right)\right)^{2}
$$

The restart stage, Step 7 works as follows. At first, the top $N_{\xi}$ vectors from the measurement step $\left\{\boldsymbol{q}_{1}, \boldsymbol{q}_{2}, \ldots, \boldsymbol{q}_{N_{\xi}}\right\}$ are scaled within the range $[0,1]$ to get $\left\{\boldsymbol{s}_{1}, \boldsymbol{s}_{2}, \ldots, \boldsymbol{s}_{N_{\xi}}\right\}$. Then the maximum Euclidean distances of the vectors centered around the best point $\boldsymbol{s}_{1}$, is computed. Finally, the distance is scaled by dividing the number of dimensions $K$.d to get

$$
\rho=\frac{\max \left\{\left\|s_{1}-s_{i}\right\|\right\}}{(K . d)}, 2 \leq i \leq N_{\xi}
$$

If $\rho$ drops below a predefined parameter $\epsilon$, an input to the algorithm, then the search is restarted. Instead of using the previously mentioned initialization, to start exploring from a different location, the restart is done by selecting $K$ number of random datapoints selected uniformly from the dataset and concatenating them to get a new vector $\boldsymbol{m}^{(0)}$ and the restarted variance $\boldsymbol{P}^{(0)}$ for the restarted process, as in Eq. (14) and (15).

Step 8 terminates the algorithm and takes $\boldsymbol{m}_{\text {best }}^{(k)}$ as a solution, if the iterations $k$ exceeds the maxiter. Else, the control goes to Step 2.

\section{Experiment}

\subsection{Used Dataset}

To test the usefulness of the proposed HKA-K and HKA clustering algorithms, we have performed experiments with two synthetic datasets and a few 
well known datasets from the UCI Machine Learning repository [3]. We have also compared the performance of HKA-K with some population based hybrid algorithms. All the algorithms were implemented with $\mathrm{C}++$ using Armadillo [37] linear algebra library. Table 1 lists the datasets used in our experiment and provides the number of points $n$, dimensionality $d$ and the number of clusters present in the data $K$.

Table 1: Datasets used for testing the clustering methods

\begin{tabular}{llllllll}
\hline & Artset1 & Artset2 & Iris & Wine & Glass & CMC & Cancer \\
\hline No. of Data: $n$ & 600 & 250 & 150 & 178 & 214 & 1473 & 683 \\
Dimensions: $d$ & 2 & 3 & 4 & 13 & 9 & 9 & 9 \\
Clusters: $K$ & 6 & 5 & 3 & 3 & 6 & 3 & 2 \\
\hline
\end{tabular}

The datasets acquired from the UCI machine learning repository listed in Table 1 were chosen because these datasets are quite frequently used in clustering related literature.The synthetic datasets Artset1 and Artset2 are generated as follows.

- Artset1 ( $n=600, d=2, K=6)$ : This two-dimensional dataset consists of 6 clusters. The plot of this dataset is shown in Figure 1a. Here 600 patterns were drawn from six independent bi-variate normal distributions, with the mean and covariances given by

$$
\begin{aligned}
& \mathcal{N}\left(\mu=\left(\begin{array}{l}
1 \\
1
\end{array}\right), \sum=\left[\begin{array}{ll}
1 & 0 \\
0 & 1
\end{array}\right]\right), \mathcal{N}\left(\mu=\left(\begin{array}{c}
5 \\
15
\end{array}\right), \sum=\left[\begin{array}{cc}
1.2 & 0 \\
0 & 1.2
\end{array}\right]\right), \\
& \mathcal{N}\left(\mu=\left(\begin{array}{c}
15 \\
-5
\end{array}\right), \sum=\left[\begin{array}{cc}
1.5 & 0 \\
0 & 1.5
\end{array}\right]\right), \mathcal{N}\left(\mu=\left(\begin{array}{l}
10 \\
10
\end{array}\right), \sum=\left[\begin{array}{cc}
1 & 0 \\
0 & 1
\end{array}\right]\right), \\
& \mathcal{N}\left(\mu=\left(\begin{array}{l}
20 \\
20
\end{array}\right), \sum=\left[\begin{array}{ll}
1 & 0 \\
0 & 1
\end{array}\right]\right), \mathcal{N}\left(\mu=\left(\begin{array}{c}
25 \\
-7
\end{array}\right), \sum=\left[\begin{array}{ll}
2 & 0 \\
0 & 2
\end{array}\right]\right) .
\end{aligned}
$$
A scatterplot for these datapoints are shown in Figure 1a,

- Artset2 ( $n=250, d=3, K=5)$ : This three-dimensional synthetic dataset consists of 5 clusters. The plot of this dataset is shown in Figure 1b. Each dimension of the dataset is class-wise distributed as Class 1 - Uniform $(85,100)$, Class 2 - Uniform(70, 85), Class 3 - Uniform $(55,70)$ Class 4 - Uniform $(40,55)$, Class 5 - Uniform $(25,40)$, where each class has 50 datapoints. A scatterplot for these datapoints are shown in Figure $1 \mathrm{~b}$. 


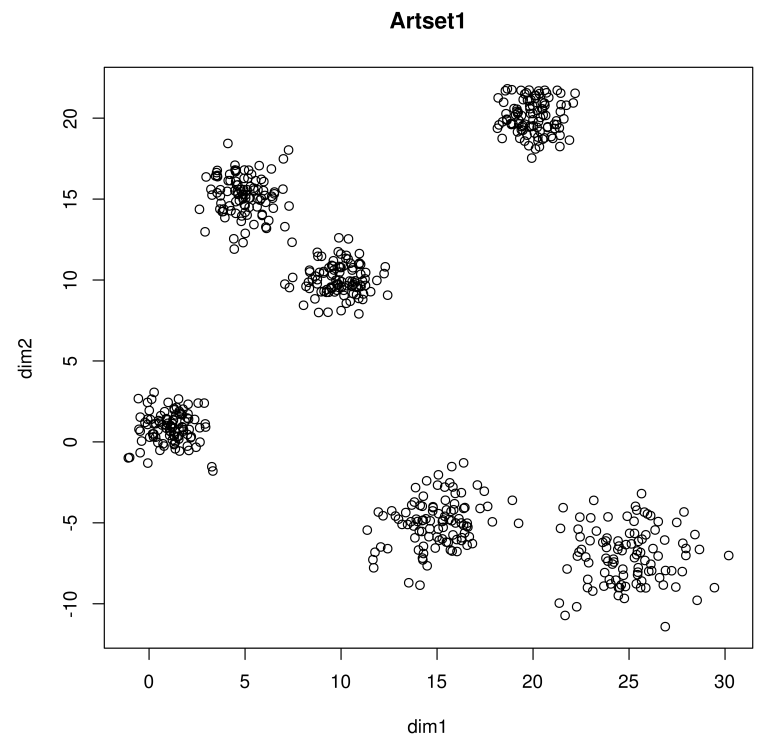

(a) Artset1

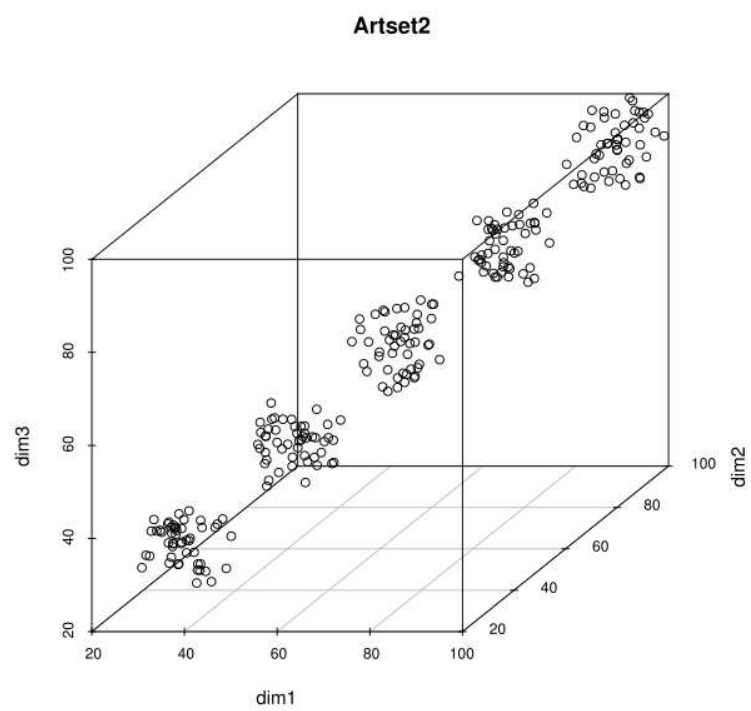

(b) Artset2

Figure 1: Synthetic Datasets 


\subsection{Used Parameters}

We have tested HKA-K and HKA clustering algorithms, as well as, KGA [27], GAC [23], ABCC [22] and PSO [6] algorithms on the above datasets and compared the results. For all the algorithms, the optimization criterion used is Eq. (12).

For conducting the experiments, the parameters for HKA clustering and HKA-K are selected as follows. At first, HKA was run on Glass dataset with different combinations of $N, N_{\xi}$ and $\alpha$, while HKA-K was run with different combinations of $\alpha, N, N_{\xi}$ and $w$. Then the parameter combination with the minimum sum of intra-cluster distances over 10 executions, (with each execution having a maximum of 250 iterations) was selected. These parameter values, given in the first two columns of Table 2 have been used for the clustering of all other datasets.

Table 2 also shows the parameters used for other four methods with which we compared our algorithm. The Tables 3 show the results for all the methods with the prescribed parameters listed in Table2, which are taken from the corresponding referenced articles. The parameters for KGA and GAC are taken from 27] (though more iterations were added to GAC). We have used the parameters for ABCC from [22], while the parameters for the PSO algorithm [6] were taken from [31] instead, as we found PSO clustering in [6] performed better with these parameters. All of the KGA, GAC, PSO, ABCC algorithms were executed 20 times with the corresponding parameters and the results are shown in Tables 3. 9. These were used to show how HKA-K and HKA clustering performs when compared to other algorithms under parameters prescribed by the authors of the corresponding algorithms. It is very important to note that the number of function evaluations highly differ to each other for each algorithms for the given selection of parameters. We have performed this test to understand the achievable cluster quality of the algorithms.

On the other hand we have performed another experiment with the parameters mentioned in Table 10 which were selected in such a way that all the algorithms approximately runs equal number of objective function evaluations. The number of function evaluations for all the algorithms are kept almost equal to HKA-K in this experiment. This is done to see how the algorithms converge when all of them evaluate approximately equal number of functions. Details are explained in Section 6.4.

Table 2: Algorithm parameters for results in Table 319

\begin{tabular}{llllll}
\hline HKA-K & HKA & KGA & GAC & ABCC & PSO \\
\hline$N=20$ & $N=30$ & pop $=50$ & pop $=50$ & $F N=20$ & pop $=10 \times K \times d$ \\
$N_{\xi}=10$ & $N_{\xi}=6$ & & & & $c 1=2.0$ \\
$\alpha=0.7$ & $\alpha=0.8$ & $p_{c}=0.8$ & $p_{c}=0.8$ & limit $=100$ & $c 2=2.0$ \\
$\epsilon=0.005$ & & $p_{m}=0.001$ & $p_{m}=0.001$ & & {$\left[w_{\text {min }}, w_{\text {max }}\right]=[0.5,1.0]$} \\
$w=0.4$ & & & & \\
maxiter $=250$ & maxiter $=500$ & gen $=1000$ & gen $=1500$ & maxiter $=1000$ & maxiter $=500$ \\
\hline
\end{tabular}

In Table 2 and Table 10, $p o p$ is the population size for KGA, GAC and 
PSO, while $p_{c}$ and $p_{m}$ are the crossover and mutation probability, respectively, for both KGA and GAC. The parameter gen indicates the number of generations the algorithms KGA and GAC are allowed to run. The parameters $c 1$ and $c 2$ are the cognitive and social factors, and the $w_{\min }$ and $w_{\max }$ are minimum and maximum momentum factors for PSO. The parameter $F N$ is the number of food sources to exploit and limit is the limit of the food source for ABCC algorithm. Also, maxiter is the number of maximum iterations taken for HKA-K, HKA clustering, ABCC and PSO algorithms.

\subsection{Evaluation Metrics}

We have used several metrics to estimate and compare the cluster quality, which are of two types, namely internal and external. The internal metrics assume that the datapoints within the clusters are close, compact and hyperspherical while different clusters are well separated, but they do not use the true class labels into consideration. On the other hand, external metrics employ actual class labels of the dataset. After the algorithm assigns the data points to the clusters, the quality is measured using the true class labels of the data.

We have used Adjusted Rand Index (ARI) [17] as an external validation index. For ARI, higher value indicates better clustering and the value 1.0 indicates best result. The ARI is computed as follows.

Let $U=\left\{\boldsymbol{u}_{1}, \boldsymbol{u}_{2}, \ldots, \boldsymbol{u}_{K}\right\}$ be the set of clusters found by the algorithm while $V=\left\{\boldsymbol{v}_{1}, \boldsymbol{v}_{2}, \ldots, \boldsymbol{v}_{K}\right\}$ defines the true class label. Let $n$ be the total number of datapoints. Also, let

- $a$ : Number of pairs of datapoints which are placed in a single cluster in $U$ and also belongs to a single class in $V$.

- $b$ : Number of pairs of datapoints which are placed in a single cluster in $U$ but belongs to different classes in $V$.

- $c$ : Number of pairs of datapoints which are placed in different clusters in $U$ but belongs to a single class in $V$.

- $d$ : Number of pairs of datapoints which are placed in different clusters in $U$ and also belongs to different classes in $V$.

Then the $A R I$ is defined as

$$
A R I=\frac{\left(\begin{array}{l}
n \\
2
\end{array}\right)(a+d)-[(a+b)(a+c)+(c+d)(b+d)]}{\left(\begin{array}{l}
n \\
2
\end{array}\right)^{2}-[(a+b)(a+c)+(c+d)(b+d)]}
$$

We have also shown the sum of intra-cluster distances (denoted as "Intra" in the tables) of the datapoints and Davies-Bouldin index [11] as internal validation metrics. Of them, the sum of intra-cluster distances (Intra) indicate the compactness of the clusters, denoted as

$$
\operatorname{Intra}(\boldsymbol{Z})=\sum_{\boldsymbol{z}_{j} \in \boldsymbol{Z}} \sum_{\boldsymbol{x}_{i} \in \boldsymbol{X}} \mu_{i j}\left\|\boldsymbol{x}_{i}-\boldsymbol{z}_{j}\right\|
$$


In Tables 3.9 the rows labeled Intra, the mean, min, max and std respectively denote the mean, minimum, maximum and the standard deviation of $\operatorname{Intra}(\boldsymbol{Z})$ over the total number of runs for the experiment. Also, the Table 11 states the mean and standard deviations of the $\operatorname{Intra}(Z)$ values.

On the other hand, Davies-Bouldin index finds the ratio of the sum of within cluster scatter to the between cluster separation. This metric assumes compact and well separated clusters as ideal. Here, a lower index value indicates better clustering. Davies-Bouldin index $D B$ is defined as:

$$
D B=\frac{1}{K} \sum_{i=1}^{K} R_{i} \text { where } R_{i}=\max _{i=1, \ldots, K}\left\{R_{i j}\right\} \quad i=1, \ldots, K ; i \neq j
$$

$R_{i j}$ is a similarity measure between clusters $\boldsymbol{c}_{i}$ and $\boldsymbol{c}_{j}$. This is computed based on the measure of dispersion $S_{i}$ and $S_{j}$ of each of $\boldsymbol{c}_{i}$ and $\boldsymbol{c}_{j}$ respectively, and the dissimilarity between two clusters $M_{i j}$, which are defined as follows:

$$
R_{i j}=\frac{S_{i}+S_{j}}{M_{i j}} ; S_{i}=\frac{1}{\left|\boldsymbol{c}_{i}\right|} \sum_{x \in \boldsymbol{c}_{i}}\left\|\boldsymbol{x}-\boldsymbol{z}_{i}\right\| ; M_{i j}=\left\|\boldsymbol{z}_{i}-\boldsymbol{z}_{j}\right\|
$$

It may be noted that in Table 3.9 we have only shown the mean values of $\mathrm{ARI}$ and DB index.

The mean execution time in seconds are also shown in Tables 3.9. The tests were run in the same system with negligible additional load. Also, the program executables were generated using the same compiler and compilation options.

\subsection{Experimental Results}

The experimental results are presented and evaluated in two ways as follows. (a) At first, we have presented clustering performance of the algorithms using the parameters prescribed in the referenced articles of the other algorithms. We have shown that HKA-K and HKA clustering can reach similar results in lesser time than the other methods. (b) Next, we have shown that the performance of our HKA-K algorithm is similar to KGA and better than other algorithms when all algorithms are allowed to run for approximately similar number of function evaluations, with nearly the same execution time.

\section{(a) Results with parameters from referenced articles}

The parameters selected for this experiment is mentioned in Table 2 and explained in Section 6.2 The results obtained by other methods as well as HKA-K and HKA clustering for different datasets are systematically shown in Table 3 9, In these tables the best results are shown in bold numerals.

At first, let us compare between HKA and HKA-K methods. From the tables it is noted that on all datasets the maximum number of iterations maxiter and the population size $N$ for HKA-K are smaller than HKA clustering method. Hence HKA-K was able to work faster and attain similar results with respect to the ARI values for Iris, Wine, Cancer and CMC dataset, and slightly better for Glass dataset, than the HKA approach. On the other hand, HKA-K was able to converge to a lower value of sum of intra cluster distance than HKA clustering. 
Next, we compare our results with those of the other methods. To be unbiased, we have used the same parameters used in the referenced articles (as given in Table 21) for our simulation. It may be observed from Table 3 19 that KGA, $\mathrm{ABCC}$ and PSO attain a similar result as HKA-K and HKA clustering, but take more computation time. For the Glass dataset, HKA-K performs better with respect to the ARI index. On the other hand, GAC was not able to reach results closer to the other algorithms with the used parameter settings. With respect to the DB index, we note that except for the Glass dataset, the values obtained by our methods are the lowest, which is desirable. For the synthetic datasets, all algorithms except GAC reached the same values on ARI and DB every time, though there was slight differences in the mean Intra value.

It is to be noted in Tables 3.9, that the executions for KGA, GAC, ABCC and PSO need several times more number of function evaluations compared to HKA-K or HKA clustering. With the parameters shown in Table 2 the approximate number of function evaluations are shown in braces as follows: KGA $(50,000)$, GAC $(75,000)$, ABCC $(40,000)$, HKA $(15,000)$, HKA-K $(5,250)$. The number of function evaluations for PSO is different for different datasets as follows: Artset1 (40,000), for Artset2 $(75,000)$, Iris $(60,000)$, Wine $(195,000)$, Glass $(270,000)$, CMC $(135,000)$, Cancer $(90,000)$. The number of function evaluations for PSO vary with respect to the datasets because the population size depends on the number of clusters and the number of dimensions of the dataset, as described in Table 2 .

The best ARI and DB index are highlighted in the tables. Also, from Tables 3 .9. for the Intra, DB and ARI index together over all datasets, the number of times the different algorithms achieved the best results are as follows: 17 times by HKA-K, 10 times by HKA clustering, 17 times by KGA, 15 times by ABCC, 7 times by PSO and 0 times by GAC. Therefore, HKA-K and KGA were able to achieve best metric values maximum number of times over all the metrics and datasets combined.

Though the trend is clear from the mean runtime values, we have performed a two-tailed Wilcoxon's rank sum test [12] for the runtimes. Wilcoxon's rank sum test is a pairwise test that detects significant difference between two sample means. The null hypothesis $H_{0}$ for the test states that, there is no significant difference between the two samples. We have taken the significance level as $\alpha=$ 0.05 for this two-tailed test. We can reject $H_{0}$ if the $p$-value for an experiment is less than the selected significance level and conclude that the difference is significant. The $p$-values for the Wilcoxon's rank sum test for HKA-K vs each of the other algorithm-dataset combination had values always less than $10^{-4}$. We could reject $H_{0}$ with the mentioned significance level, as the $p$-values were much below 0.05 . 
Table 3: Results for Artset1

\begin{tabular}{|c|c|c|c|c|c|c|c|c|}
\hline Dataset & Validity & & HKA-K & HKA & KGA & GAC & $\mathrm{ABCC}$ & PSO \\
\hline \multirow{7}{*}{ Artset1 } & \multirow{4}{*}{ Intra } & mean & 918.1220 & 954.2652 & 918.1220 & 5630.5230 & 918.1235 & 918.1226 \\
\hline & & std & 0.0000 & 142.2113 & 0.0000 & 132.9182 & 0.0150 & 0.0027 \\
\hline & & $\min$ & 918.1220 & 918.0910 & 918.1220 & 5358.7400 & 918.1050 & 918.1180 \\
\hline & & $\max$ & 918.1220 & 1554.2600 & 918.1220 & 5815.2300 & 918.1550 & 918.1290 \\
\hline & ARI & \multirow{2}{*}{ mean } & 0.9960 & 0.9844 & 0.9960 & 0.1234 & 0.9960 & 0.9960 \\
\hline & $\overline{\mathrm{DB}}$ & & 0.3204 & 0.3347 & 0.3204 & 7.1917 & 0.3204 & 0.3204 \\
\hline & Time (sec) & mean & 0.3993 & 1.0327 & 11.5204 & 6.6328 & 2.5441 & 2.7118 \\
\hline
\end{tabular}

Table 4: Results for Artset2

\begin{tabular}{|c|c|c|c|c|c|c|c|c|}
\hline Dataset & Validity & & HKA-K & HKA & KGA & GAC & $\overline{\mathrm{ABCC}}$ & $\mathrm{PSO}$ \\
\hline \multirow{7}{*}{ Artset2 } & \multirow{4}{*}{ Intra } & mean & 1782.1000 & 1782.0920 & 1782.1000 & 3906.7310 & 1782.0770 & 1782.2160 \\
\hline & & std & 0.0000 & 0.0106 & 0.0000 & 220.8353 & 0.0330 & 0.2911 \\
\hline & & $\min$ & 1782.1000 & 1782.0700 & 1782.1000 & 3605.4100 & 1781.9900 & 1781.8200 \\
\hline & & $\max$ & 1782.1000 & 1782.1100 & 1782.1000 & 4305.4900 & 1782.1000 & 1782.8800 \\
\hline & ARI & \multirow{2}{*}{ mean } & 1.0000 & 1.0000 & 1.0000 & 0.3080 & 1.0000 & 1.0000 \\
\hline & $\overline{\mathrm{DB}}$ & & 0.5593 & 0.5593 & 0.5593 & 3.2681 & 0.5593 & 0.5593 \\
\hline & Time (sec) & mean & 0.1985 & 0.4537 & 6.8196 & 4.6298 & 1.1767 & 2.7357 \\
\hline
\end{tabular}

Table 5: Results for Iris dataset

\begin{tabular}{l|l|lll|llll}
\hline Dataset & Validity & & HKA-K & HKA & KGA & GAC & ABCC & PSO \\
\hline \multirow{4}{*}{ Iris } & & mean & $\mathbf{9 7 . 3 2 5 9}$ & 97.3289 & $\mathbf{9 7 . 3 2 5 9}$ & 101.2907 & 97.3262 & 97.3320 \\
& & std & 0.0000 & 0.0096 & 0.0000 & 2.3798 & 0.0012 & 0.0335 \\
& & min & 97.3259 & 97.3129 & 97.3259 & 97.7973 & 97.325 & 97.3258 \\
& & max & 97.3259 & 97.3512 & 97.3259 & 105.1990 & 97.3295 & 97.3471 \\
\cline { 2 - 9 } & ARI & \multirow{2}{*}{ mean } & $\mathbf{0 . 7 3 0 2}$ & $\mathbf{0 . 7 3 0 2}$ & $\mathbf{0 . 7 3 0 2}$ & 0.7171 & $\mathbf{0 . 7 3 0 2}$ & 0.7261 \\
\cline { 2 - 9 } & DB & & $\mathbf{0 . 6 6 2 3}$ & $\mathbf{0 . 6 6 2 3}$ & $\mathbf{0 . 6 6 2 3}$ & 0.7446 & $\mathbf{0 . 6 6 2 3}$ & 0.6635 \\
\cline { 2 - 8 } & Time (sec) & mean & $\mathbf{0 . 0 7 7 8}$ & 0.2281 & 3.2496 & 4.5995 & 0.5436 & 0.6379 \\
\hline
\end{tabular}

Table 6: Results for Wine dataset

\begin{tabular}{|c|c|c|c|c|c|c|c|c|}
\hline Dataset & Validity & & HKA-K & HKA & KGA & GAC & $\mathrm{ABCC}$ & PSO \\
\hline \multirow{7}{*}{ Wine } & \multirow{4}{*}{ Intra } & mean & 16555.7000 & 16574.0800 & 16555.7000 & 18418.2200 & 16557.4200 & 16557.4100 \\
\hline & & std & 0.0000 & 8.3174 & 0.0000 & 637.6402 & 17.2601 & 0.7564 \\
\hline & & $\min$ & 16555.7000 & 16564.2000 & 16555.7000 & 17369.4000 & 16542.3000 & 16556.5000 \\
\hline & & $\max$ & 16555.7000 & 16595.0000 & 16555.7000 & 19665.9000 & 16569.1000 & 16558.6000 \\
\hline & ARI & \multirow{2}{*}{ mean } & 0.3711 & 0.3711 & 0.3711 & 0.3679 & 0.3711 & 0.3711 \\
\hline & $\overline{\mathrm{DB}}$ & & 0.5342 & 0.5342 & 0.5342 & 0.6597 & 0.5342 & 0.5342 \\
\hline & Time (sec) & mean & 0.2010 & 0.578 & 5.1260 & 5.7110 & 1.3319 & 1.6246 \\
\hline
\end{tabular}

Table 7: Results for Glass dataset

\begin{tabular}{|c|c|c|c|c|c|c|c|c|}
\hline Dataset & Validity & & HKA-K & HKA & $\overline{\mathrm{KGA}}$ & GAC & ABCC & PSO \\
\hline \multirow{7}{*}{ Glass } & & mean & 215.5866 & 216.4745 & 215.4700 & 288.8954 & 216.1428 & 216.0280 \\
\hline & Int & std & 0.5211 & 1.3169 & 0.0000 & 13.3002 & 1.4826 & 2.6959 \\
\hline & intra & $\min$ & 213.4210 & 215.7260 & 215.4700 & 273.1690 & 213.7490 & 214.2650 \\
\hline & & $\max$ & 215.9210 & 219.7340 & 215.4700 & 318.1390 & 221.4760 & 219.6710 \\
\hline & ARI & \multirow{2}{*}{ mean } & 0.2664 & 0.2645 & 0.2552 & 0.1266 & 0.2583 & 0.2556 \\
\hline & $\overline{\mathrm{DB}}$ & & 0.9556 & 0.9755 & 0.9351 & 4.7682 & 0.9092 & 0.9174 \\
\hline & Time (sec) & mean & 0.3233 & 0.8469 & 7.0956 & 6.4254 & 2.1436 & 16.1743 \\
\hline
\end{tabular}


Table 8: Results for CMC dataset

\begin{tabular}{l|l|lll|llll}
\hline Dataset & \multicolumn{1}{l}{ Validity } & & HKA-K & HKA & KGA & GAC & ABCC & PSO \\
\hline \multirow{5}{*}{ CMC } & & mean & 5545.0500 & 5545.2620 & 5545.0500 & 10051.9040 & $\mathbf{5 5 4 5 . 0 6 3 0}$ & 5545.7160 \\
& Intra & std & 0.0000 & 0.2267 & 0.0000 & 145.9788 & 0.0747 & 7.1344 \\
& & min & 5545.0500 & 5544.7700 & 5545.0500 & 9729.1200 & 5544.9900 & 5545.2000 \\
& & max & 5545.0500 & 5545.6400 & 5545.0500 & 10212.3000 & 5545.1900 & 5546.3300 \\
\cline { 2 - 9 } & ARI & \multirow{2}{*}{ mean } & $\mathbf{0 . 0 2 6 0}$ & $\mathbf{0 . 0 2 6 0}$ & $\mathbf{0 . 0 2 6 0}$ & 0.0052 & $\mathbf{0 . 0 2 6 0}$ & 0.0259 \\
\cline { 2 - 9 } & DB & & $\mathbf{0 . 7 6 6 3}$ & $\mathbf{0 . 7 6 6 3}$ & $\mathbf{0 . 7 6 6 3}$ & 4.9677 & 0.7672 & $\mathbf{0 . 7 6 6 3}$ \\
\cline { 2 - 9 } & Time (sec) & mean & $\mathbf{1 . 1 7 2 4 4 6}$ & 3.1515 & 21.2861 & 16.7148 & 8.2142 & 38.9426 \\
\hline
\end{tabular}

Table 9: Results for Cancer dataset

\begin{tabular}{c|l|lll|llll}
\hline \multicolumn{1}{l}{ Dataset } & \multicolumn{1}{l}{ Validity } & HKA-K & HKA & KGA & GAC & ABCC & PSO \\
\hline \multirow{5}{*}{ Cancer } & & mean & $\mathbf{2 9 8 8 . 4 3 0 0}$ & 2988.4390 & $\mathbf{2 9 8 8 . 4 3 0 0}$ & 3962.1350 & $\mathbf{2 9 8 8 . 4 3 0 0}$ & 2988.4540 \\
& & std & 0.0000 & 0.1353 & 0.0000 & 59.1108 & 0.0000 & 0.0479 \\
& & min & 2988.4300 & 2988.1100 & 2988.4300 & 3878.3700 & 2988.4300 & 2988.4000 \\
& & max & 2988.4300 & 2988.6600 & 2988.4300 & 4072.0300 & 2988.4300 & 2988.5300 \\
\cline { 2 - 8 } & ARI & \multirow{2}{*}{ mean } & $\mathbf{0 . 8 4 6 5}$ & $\mathbf{0 . 8 4 6 5}$ & $\mathbf{0 . 8 4 6 5}$ & 0.4159 & $\mathbf{0 . 8 4 6 5}$ & $\mathbf{0 . 8 4 6 5}$ \\
\cline { 2 - 9 } & DB & & $\mathbf{0 . 7 5 7 3}$ & $\mathbf{0 . 7 5 7 3}$ & $\mathbf{0 . 7 5 7 3}$ & 1.2892 & $\mathbf{0 . 7 5 7 3}$ & $\mathbf{0 . 7 5 7 3}$ \\
\cline { 2 - 9 } & Time (sec) & mean & $\mathbf{0 . 4 2 2 8}$ & 1.1768 & 9.2882 & 8.9919 & 3.1528 & 3.7636 \\
\hline
\end{tabular}

(b) Results with equal number of function evaluations

The parameters selected in the previous experiment for KGA, GAC, ABCC and PSO from the referenced articles are mentioned in Section 6.2. The numbers of function evaluations for these selections differ substantially from each other and are much larger than what HKA-K and HKA clustering use, as discussed before.

To show how the algorithms converge when they are allowed to run for approximately equal number of function evaluations, we perform another experiment by selecting the parameters of the other algorithms in such a way that it approximately matches the number of function evaluations for HKA-K, which is 5250 in this case. The parameters selected for this experiment are mentioned in Table 10 and explained in Section 6.2. Table 11 shows the mean and standard deviation of the $\operatorname{Intra}(Z)$ values computed over 20 runs for each algorithm-dataset combination for parameters given in Table 10, Note that Table 10 shows the parameters for each algorithm such that each of them performs around 6000 function evaluations. From Table 11 it can be seen that HKA-K has converged to a better value of $\operatorname{Intra}(Z)$ than HKA and GAC, ABCC, PSO algorithms for a similar number of function evaluations. On the other hand, KGA was able to converge as good as HKA-K.

Table 10: Algorithm parameters for results in Table 11]

\begin{tabular}{llllll}
\hline HKA-K & HKA & KGA & GAC & ABCC & PSO \\
\hline$N=20$ & $N=20$ & pop $=12$ & pop $=12$ & $F N=12$ & pop $=12$ \\
$N_{\xi}=10$ & $N_{\xi}=10$ & & & & $c 1=2.0$ \\
$\alpha=0.7$ & $\alpha=0.8$ & $p_{c}=0.8$ & $p_{c}=0.8$ & limit $=15$ & $c 2=2.0$ \\
$\epsilon=0.005$ & & $p_{m}=0.001$ & $p_{m}=0.001$ & & {$\left[w_{\text {min }}, w_{\text {max }}\right]=[0.5,1.0]$} \\
$w=0.4$ & & & & & \\
maxiter $=250$ & maxiter $=250$ & gen $=250$ & gen $=250$ & maxiter $=250$ & maxiter $=500$ \\
\hline
\end{tabular}


Table 11: Intra $(Z)$ values for algorithms on datasets for similar numbers of function evaluations using parameters from Table 10

\begin{tabular}{llll|llll}
\hline & Intra $(Z)$ & HKA-K & HKA & KGA & GAC & ABCC & PSO \\
\hline \multirow{2}{*}{ Artset1 } & mean & $\mathbf{9 1 8 . 1 2 2 0}$ & 1758.8000 & $\mathbf{9 1 8 . 1 2 2 0}$ & 6983.3830 & 969.9842 & 967.4472 \\
& std & 0.00 & 815.33 & 0.00 & 75.50 & 25.50 & 26.45 \\
\hline \multirow{2}{*}{ Artset2 } & mean & $\mathbf{1 7 8 2 . 1 0 0 0}$ & 1823.1655 & 1793.1070 & 6751.3695 & 1839.6760 & 1783.3710 \\
& std & 0.00 & 153.01 & 37.04 & 176.01 & 39.74 & 1.11 \\
\hline \multirow{2}{*}{ Iris } & mean & $\mathbf{9 7 . 3 2 5 9}$ & 97.3537 & $\mathbf{9 7 . 3 2 5 9}$ & 226.2000 & 97.6008 & 98.7280 \\
& std & 0.00 & 0.04 & 0.00 & 9.89 & 0.34 & 0.82 \\
\hline \multirow{2}{*}{ Wine } & mean & $\mathbf{1 6 5 5 5 . 7 0 0 0}$ & 17066.4850 & $\mathbf{1 6 5 5 5 . 7 0 0 0}$ & 36260.9500 & 16688.7450 & 16654.0500 \\
& std & 0.00 & 391.70 & 0.00 & 1476.84 & 196.42 & 56.40 \\
\hline \multirow{2}{*}{ Glass } & mean & $\mathbf{2 1 5 . 7 0 2 1}$ & 223.9402 & 229.7236 & 397.2842 & 269.1553 & 229.3859 \\
& std & 0.85 & 6.13 & 18.05 & 8.71 & 17.24 & 3.91 \\
\hline \multirow{2}{*}{ CMC } & mean & 5545.0500 & 5617.8940 & $\mathbf{5 5 4 4 . 9 9 8 5}$ & 11141.6650 & 5570.1785 & 5676.7885 \\
& std & 0.00 & 20.22 & 0.83 & 36.97 & 23.16 & 37.20 \\
\hline \multirow{2}{*}{ Cancer } & mean & $\mathbf{2 9 8 8 . 4 3 0 0}$ & 3667.6215 & $\mathbf{2 9 8 8 . 4 3 0 0}$ & 4927.9800 & 2996.6850 & 3137.9700 \\
& std & 0.00 & 201.83 & 0.00 & 33.85 & 13.73 & 0.00 \\
\hline
\end{tabular}

Table 12: HKA-K vs All, Wilcoxon's Rank Sum test p-values, Level of significance $\alpha=0.025$. For the underlined values $H_{0}$ could not be rejected. For other values $H_{0}$ was rejected.

\begin{tabular}{llllll}
\hline HKA-K vs & HKA & KGA & GAC & ABCC & PSO \\
\hline Artset1 & $1.907 \times 10^{-6}$ & $\underline{1.0000}$ & $9.537 \times 10^{-7}$ & $9.537 \times 10^{-7}$ & $9.537 \times 10^{-7}$ \\
Artset2 & $1.907 \times 10^{-6}$ & $\underline{\underline{1.0000}}$ & $9.537 \times 10^{-7}$ & $9.537 \times 10^{-7}$ & $9.537 \times 10^{-7}$ \\
Iris & 0.003648 & $\underline{\underline{1.0000}}$ & $9.537 \times 10^{-7}$ & 0.0002413 & $9.537 \times 10^{-7}$ \\
Wine & $9.537 \times 10^{-7}$ & $\underline{\underline{1.0000}}$ & $9.537 \times 10^{-7}$ & 0.00243 & $9.537 \times 10^{-7}$ \\
Glass & $9.537 \times 10^{-7}$ & $\underline{0.0003} 175$ & $9.537 \times 10^{-7}$ & $9.537 \times 10^{-7}$ & $9.537 \times 10^{-7}$ \\
CMC & $4.778 \times 10^{-5}$ & $\underline{0.3051}$ & $9.537 \times 10^{-7}$ & $4.778 \times 10^{-5}$ & $9.537 \times 10^{-7}$ \\
Cancer & $9.537 \times 10^{-5}$ & $\underline{1.0000}$ & $9.537 \times 10^{-7}$ & 0.0265 & $9.537 \times 10^{-7}$ \\
\hline
\end{tabular}


To understand if HKA-K results were significantly better than the other algorithms we conduct a one-tailed Wilcoxon's rank sum test for this experiment. The test is done with HKA-K vs all other algorithms for each of the datasets with respect to the $\operatorname{Intra}(Z)$ values for this experiment. The null hypothesis $H_{0}$ is the same as defined before. The alternative hypothesis is, HKA-K has a lower Intra(Z) value than the same of the compared algorithm. The level of significance is kept as $\alpha=0.025$ for this one-tailed test. The results are shown in Table 12. The values in the cells of the Table 12 are the $p$-values resulted from a one-tailed Wilcoxon's rank sum test with HKA-K vs the algorithm in the corresponding column, for the dataset in the corresponding row. The underlined values in the table are those which has a $p$-value greater than selected significance level $\alpha=0.025$. We can reject $H_{0}$ for the experiments for which the

$p$-value is less than $\alpha$, thus indicating the difference is significant. If the $p$-value is greater than or equal to $\alpha$, then we cannot make any definite conclusion. Therefore, for the underlined values, we cannot reject $H_{0}$ and make any definite conclusion.

The Tables 11 and 12 indicate that HKA-K is better than all, except KGA, for which we could not reject the null hypothesis $H_{0}$ for any datasets except Glass. For the Cancer dataset, HKA-K had a lower Intra(Z) value than that of $\mathrm{ABCC}$, but the difference was not significant as the p-values were marginal. In other words, HKA-K is as good as KGA and neither could decisively outperform each other. For the other algorithms they reached a worse result in using the same number of function evaluations. The similar kind of results for HKA-K and KGA is because both of them uses a K-Means like operator, which guides the solution to a better point quickly. Although both of them are prone to converge to a local optimum. KGA avoids it by the mutation operator, while HKA-K avoids it with the conditional restart step and a carefully selected value of $w$.

This shows that, given a similar number of function evaluations, the convergence of HKA-K is significantly better than HKA, GAC, ABCC and PSO for all the datasets and better than KGA with respect to the Glass dataset. Although HKA-K does not have a significantly different performance compared to KGA, indicating both perform nearly the same.

\section{Discussion and Conclusion}

The objective of this work was to demonstrate that clustering can be performed in the HKA framework and its improvement can be another state-of-theart approach in data clustering. By testing on benchmark datasets we showed that HKA-K can achieve clustering as good as the compared algorithms with respect to internal and external validation metrics in lesser time and substantially lesser number of function evaluations. We have also shown HKA-K was able to converge to a significantly better value than the other compared algorithms except KGA, when they all run for similar number of function evaluations. KGA and HKA-K performances are of similar quality. 
There is a basic difference between our method and other clustering methods we have compared with. Though the approach in this paper is also a population based method, only one solution per iteration is generated, which approaches to an optimal point, as mentioned in Section 3 . For the other algorithms each point of the population represents a possible solution, which moves within the search space, and is kept in the memory. Whereas for our approach, the population generated using the Gaussian pdf guided by its mean and the covariance matrix, which together determines a single solution for an iteration, which is only kept in the memory. This may lead to a premature convergence of the algorithm. We have attempted to overcome this by using the conditional restart stage.

As HKA-K uses one step of K-Means as described in Section 5, the weight for this operator as well as the other parameters has to be determined in such a way that it does not lead to a premature convergence or does not spend too much time to search. Although we have set values for the parameters through a data driven procedure, it may not work equally well for all kinds of data sets.

In future it would be interesting to investigate other hybridisations with HKA. A study to improve the performance of HKA as a metaheuristic population based optimization algorithm can be investigated too. Also, a new and ingenious multi-solution framework can be devised for this proposed methodology in place of the conditional restart method.

Acknowledgements: We sincerely thank the unknown reviewers for their constructive comments to improve the quality of the paper.

\section{References}

[1] A. Afshar, O. B. Haddad, M. Marino, B. Adams, Honey-bee mating optimization (HBMO) algorithm for optimal reservoir operation, Journal of the Franklin Institute 344 (5) (2007) 452 - 462, modeling, Simulation and Applied Optimization Part II.

URL/http://www.sciencedirect.com/science/article/pii/S0016003206000822

[2] A. Ahmadyfard, H. Modares, Combining PSO and k-means to enhance data clustering, in: Telecommunications, 2008. IST 2008. International Symposium on, 2008.

[3] K. Bache, M. Lichman, UCI machine learning repository (2013). URL http://archive.ics.uci.edu/ml

[4] C. M. Bishop, Pattern Recognition and Machine Learning (Information Science and Statistics), Springer-Verlag New York, Inc., Secaucus, NJ, USA, 2006.

[5] T. Caliński, J. Harabasz, A dendrite method for cluster analysis, Communications in Statistics 3 (1) (1974) 1-27. URL http://dx.doi.org/10.1080/03610927408827101 
[6] C.-Y. Chen, F. Ye, Particle swarm optimization algorithm and its application to clustering analysis, in: Networking, Sensing and Control, 2004 IEEE International Conference on, vol. 2, 2004.

[7] Y. Cheng, M. Jiang, D. Yuan, Novel clustering algorithms based on improved artificial fish swarm algorithm, in: Fuzzy Systems and Knowledge Discovery, 2009. FSKD '09. Sixth International Conference on, vol. 3, 2009.

[8] A. Chowdhury, S. Bose, S. Das, Automatic clustering based on invasive weed optimization algorithm, in: B. Panigrahi, P. Suganthan, S. Das, S. Satapathy (eds.), Swarm, Evolutionary, and Memetic Computing, vol. 7077 of Lecture Notes in Computer Science, Springer Berlin Heidelberg, 2011, pp. $105-112$.

URL http://dx.doi.org/10.1007/978-3-642-27242-4_13

[9] M. Cowgill, R. Harvey, L. Watson, A genetic algorithm approach to cluster analysis, Computers \& Mathematics with Applications 37 (7) (1999) 99 108. URL http://www.sciencedirect.com/science/article/pii/S0898122199000905

[10] X. Cui, T. E. Potok, Document clustering analysis based on hybrid PSO $+\mathrm{k}-$ means algorithm, Special Issue (2005) 27-33.

[11] D. L. Davies, D. W. Bouldin, A cluster separation measure, Pattern Analysis and Machine Intelligence, IEEE Transactions on PAMI-1 (2) (1979) $224-227$.

[12] J. Derrac, S. García, D. Molina, F. Herrera, A practical tutorial on the use of nonparametric statistical tests as a methodology for comparing evolutionary and swarm intelligence algorithms, Swarm and Evolutionary

Computation 1 (1) (2011) 3 - 18.

URL http://www.sciencedirect.com/science/article/pii/S2210650211000034

[13] R. Faragher, Understanding the basis of the kalman filter via a simple and intuitive derivation [lecture notes], Signal Processing Magazine, IEEE 29 (5) (2012) 128-132.

[14] M. Fathian, B. Amiri, A. Maroosi, Application of honey-bee mating optimization algorithm on clustering, Applied Mathematics and Computation 190 (2) (2007) $1502-1513$.

URL http://www.sciencedirect.com/science/article/pii/S0096300307001853

[15] A. Hatamlou, Black hole: A new heuristic optimization approach for data clustering, Information Sciences 222 (0) (2013) 175 - 184, including Special Section on New Trends in Ambient Intelligence and Bio-inspired Systems. URL http://www.sciencedirect.com/science/article/pii/S0020025512005762

[16] A. Hatamlou, S. Abdullah, H. Nezamabadi-pour, A combined approach for clustering based on k-means and gravitational search algorithms, Swarm 
and Evolutionary Computation 6 (0) (2012) $47-52$.

URL http://www.sciencedirect.com/science/article/pii/S2210650212000235

[17] L. Hubert, P. Arabie, Comparing partitions, Journal of Classification 2 (1) (1985) 193-218.

URL http://dx.doi.org/10.1007/BF01908075

[18] A. K. Jain, M. N. Murty, P. J. Flynn, Data clustering: A review, ACM Comput. Surv. 31 (3) (1999) 264-323.

URL http://doi .acm.org/10.1145/331499.331504

[19] Y.-T. Kao, E. Zahara, I.-W. Kao, A hybridized approach to data clustering, Expert Systems with Applications 34 (3) (2008) $1754-1762$.

URL http://www.sciencedirect.com/science/article/pii/S0957417407000528

[20] D. Karaboga, B. Basturk, A powerful and efficient algorithm for numerical function optimization: Artificial bee colony (abc) algorithm, J. of Global Optimization 39 (3) (2007) 459-471.

URL http://dx.doi.org/10.1007/s10898-007-9149-x

[21] D. Karaboga, B. Basturk, On the performance of artificial bee colony (abc) algorithm, Applied Soft Computing 8 (1) (2008) 687 - 697. URL http://www.sciencedirect.com/science/article/pii/S1568494607000531

[22] D. Karaboga, C. Ozturk, A novel clustering approach: Artificial bee colony (ABC) algorithm, Applied Soft Computing 11 (1) (2011) 652 - 657. URL http://www.sciencedirect.com/science/article/pii/S1568494609002798

[23] K. Krishna, M. Murty, Genetic k-means algorithm, Systems, Man, and Cybernetics, Part B: Cybernetics, IEEE Transactions on 29 (3) (1999) 433-439.

[24] R. Kuo, Y. Syu, Z.-Y. Chen, F. Tien, Integration of particle swarm optimization and genetic algorithm for dynamic clustering, Information Sciences 195 (0) (2012) $124-140$.

URL http://www.sciencedirect.com/science/article/pii/S0020025512000400

[25] R. J. Kuo, L. M. Lin, Application of a hybrid of genetic algorithm and particle swarm optimization algorithm for order clustering, Decis. Support Syst. 49 (4) (2010) 451-462.

URL http://dx.doi.org/10.1016/j.dss.2010.05.006

[26] W. Kwedlo, A clustering method combining differential evolution with the k-means algorithm, Pattern Recognition Letters 32 (12) (2011) 1613 - 1621. URL http://www.sciencedirect.com/science/article/pii/S0167865511001541

[27] U. Maulik, S. Bandyopadhyay, Genetic algorithm-based clustering technique, Pattern Recognition 33 (9) (2000) 1455 - 1465.

URL http://www.sciencedirect.com/science/article/pii/S0031320399001375 
[28] P. S. Maybeck, Stochastic models, estimation, and control, vol. 141 of Mathematics in Science and Engineering, 1979.

[29] C. Murthy, N. Chowdhury, In search of optimal clusters using genetic algorithms, Pattern Recognition Letters 17 (8) (1996) 825 - 832. URL http://www.sciencedirect.com/science/article/pii/0167865596000438

[30] S. J. Nanda, G. Panda, A survey on nature inspired metaheuristic algorithms for partitional clustering, Swarm and Evolutionary Computation 16 (0) (2014) $1-18$. URL http://www.sciencedirect.com/science/article/pii/S221065021300076X

[31] T. Niknam, B. Amiri, An efficient hybrid approach based on PSO, ACO and k-means for cluster analysis, Applied Soft Computing 10 (1) (2010) $183-197$. URL http://wWw.sciencedirect.com/science/article/pii/S1568494609000854

[32] T. Niknam, B. B. Firouzi, M. Nayeripour, An efficient hybrid evolutionary algorithm for cluster analysis, World Applied Sciences Journal 4 (2) (2008) 300-307.

URL http://www.idosi.org/wasj/wasj4(2)/23.pdf

[33] T. Niknam, J. Olamaei, B. Amiri, A hybrid evolutionary algorithm based on ACO and SA for cluster analysis, Journal of Applied Sciences 8 (2008) $2695-2702$.

[34] S. Rana, S. Jasola, R. Kumar, A boundary restricted adaptive particle swarm optimization for data clustering, International Journal of Machine Learning and Cybernetics 4 (4) (2013) 391-400. URL http://dx.doi.org/10.1007/s13042-012-0103-y

[35] E. Rashedi, H. Nezamabadi-pour, S. Saryazdi, GSA: A gravitational search algorithm, Information Sciences 179 (13) (2009) 2232 - 2248, special Section on High Order Fuzzy Sets. URL http://www.sciencedirect.com/science/article/pii/S0020025509001200

[36] I. B. Saida, K. Nadjet, B. Omar, A new algorithm for data clustering based on cuckoo search optimization, in: J.-S. Pan, P. Krömer, V. Snášel (eds.), Genetic and Evolutionary Computing, vol. 238 of Advances in Intelligent Systems and Computing, Springer International Publishing, 2014, pp. 5564 .

URL http://dx.doi.org/10.1007/978-3-319-01796-9_6

[37] C. Sanderson, Armadillo: An open source $\mathrm{C}++$ linear algebra library for fast prototyping and computationally intensive experiments, Tech. rep., NICTA, Australia (October 2010).

[38] S. Z. Selim, M. A. Ismail, K-means-type algorithms: A generalized convergence theorem and characterization of local optimality, Pattern Analysis and Machine Intelligence, IEEE Transactions on PAMI-6 (1) (1984) 81-87. 
[39] P. Shelokar, V. Jayaraman, B. Kulkarni, An ant colony approach for clustering, Analytica Chimica Acta 509 (2) (2004) 187 - 195. URL http://www.sciencedirect.com/science/article/pii/S0003267003016374

[40] C. Sung, H. Jin, A tabu-search-based heuristic for clustering, Pattern Recognition 33 (5) (2000) 849 - 858. URL http://www.sciencedirect.com/science/article/pii/S0031320399000904

[41] R. Toscano, P. Lyonnet, A new heuristic approach for non-convex optimization problems, Information Sciences 180 (10) (2010) 1955 - 1966, special Issue on Intelligent Distributed Information Systems. URL http://www.sciencedirect.com/science/article/pii/S0020025509005714

[42] R. Toscano, P. Lyonnet, A kalman optimization approach for solving some industrial electronics problems, Industrial Electronics, IEEE Transactions on 59 (11) (2012) 4456-4464.

[43] D. W. Van Der Merwe, A. Engelbrecht, Data clustering using particle swarm optimization, in: Evolutionary Computation, 2003. CEC '03. The 2003 Congress on, vol. 1, 2003.

[44] M. Wan, L. Li, J. Xiao, C. Wang, Y. Yang, Data clustering using bacterial foraging optimization, Journal of Intelligent Information Systems 38 (2) (2012) 321-341.

URL http://dx.doi.org/10.1007/s10844-011-0158-3

[45] K.-L. Wu, M.-S. Yang, Alternative c-means clustering algorithms, Pattern Recognition 35 (10) (2002) 2267 - 2278. URL http://www.sciencedirect.com/science/article/pii/S0031320301001972

[46] F. Ye, C.-Y. Chen, Alternative KPSO-clustering algorithm, Tamkang Journal of Science and Engineering 8 (2) (2005) 165 - 174.

[47] C. Zhang, D. Ouyang, J. Ning, An artificial bee colony approach for clustering, Expert Systems with Applications 37 (7) (2010) 4761 - 4767. URL http://www.sciencedirect.com/science/article/pii/S0957417409009452

[48] W. Zhu, J. Jiang, C. Song, L. Bao, Clustering algorithm based on fuzzy c-means and artificial fish swarm, Procedia Engineering 29 (0) (2012) 3307 - 3311, 2012 International Workshop on Information and Electronics Engineering. URL http://www.sciencedirect.com/science/article/pii/S187770581200495X 